\title{
CARDIOVASCULAR RISK AND ALL-CAUSE MORTALITY; A 12 YEAR FOLLOW-UP STUDY IN THE NETHERLANDS
}

\author{
A.W. HOES* ${ }^{* * 1}$, D.E. GROBBEE*, H.A. VALKENBURG*, J. LUBSEN*** \\ and A. HOFMAN* \\ *Department of Epidemiology \& Biostatistics - Erasmus University Medical School - P.O. Box 1738 \\ 3000 DR - Rotterdam - The Netherlands. \\ **Department of General Practice - Erasmus University Medical School - Rotterdam \\ The Netherlands. \\ ***Thoraxcenter - Academic Hospital Dijkzigt - Erasmus University Medical School - Rotterdam \\ The Netherlands.
}

Key words: Cardiovascular risk - All-cause mortality - Risk function

To assess the contribution of cardiovascular risk indicators to all-cause mortality, we used data from a follow-up study conducted in the Netherlands since 1975. Of 6,057 participants aged 20 years or over at the start of the study, $9.5 \%$ died during the 9 to 12 year follow-up period. Risk indicators independently related to all-cause mortality were age and diabetes mellitus in both sexes; pulse rate, smoking habits, antihypertensive drug use and a history of myocardial infarction most clearly in men; and body mass index and systolic blood pressure in women. A larger body mass index was associated with a gradual decrease in mortality probability. The risk of death for women in the highest quartile of body mass index $\left(>26.4 \mathrm{~kg} / \mathrm{m}^{2}\right)$ relative to those in the lowest quartile $(<21.9 \mathrm{~kg} /$ $\mathrm{m}^{2}$ ) was $0.56(95 \%$ confidence limits 0.36 and 0.87$)$. Serum cholesterol level showed no association with overall mortality.

Risk functions were calculated to predict an individual's probability of dying within 11.5 years as a function of the level of cardiovascular risk indicators.

Our findings suggest that the major cardiovascular risk indicators apart from affecting cardiovascular morbidity and mortality, also influence all-cause mortality. Consequently, favourable changes in these charateristics might lead to an increase in life expectancy. The maximum individual benefit to be expected from these changes may be estimated using the risk functions derived from our data.

\section{INTRODUCTION}

Many longitudinal studies have produced evidence for an association of certain risk indicators, e.g. blood pressure, smoking and serum cholesterol, with cardiovascular morbidity and mortality $(1,14,15$, $26,27,34)$. In recent years more emphasis has been put on the contribution of these cardiovascular risk indicators in predicting death from all causes $(18,21$, $22,24,25)$. The latter approach offers certain advantages. The identification of risk indicators of all-

\footnotetext{
Corresponding author.
}

cause mortality would focus on preventive measures aimed at improving the primary health parameter of a population: life expectancy. Measures restricted to risk indicators of a specific disease category may simultaneously enhance the chance of developing another disease and hence have no effect on, or even be detrimental to survival. Moreover, individuals may be more interested in the impact of changes in their cardiovascular risk profile on survival, than on their chance of experiencing a specific event.

Once identified, these risk indicators may be used to obtain risk functions that estimate the probability of occurrence of an event, in this case death, as function 
of the level of the risk indicators (14). This approach not only enables estimation of an individual's probability of dying within a certain time span, but also allows calculation of the maximum benefit to be expected from risk factor intervention.

We studied the contribution of cardiovascular risk indicators to all-cause mortality, using data from a large follow-up study conducted since the midseventies in the Netherlands. Further, risk functions predicting the 11.5 years probability of death were derived.

\section{MATERIALS AND METHODS}

\section{Subjects}

Between 1975 and 1978 a study was initiated in Zoetermeer, a town with at that time 60,000 inhabitants in the western part of the Netherlands (31). The objective was to study cardiovascular and other chronic diseases and their determinants. All 13,462 inhabitants aged 5 years or over of two districts were invited to participate. Only those above 20 years of age were included in the present analysis. The response in this age group was $75.6 \%(n=6,547)$.

At entry into the study the participants were interviewed by a physician, a venous blood sample and a urine sample was obtained, a self-administered questionnaire was checked, and a brief physical examination, including measurements of blood pressure pulse rate and body mass index, was performed. The cardiovascular risk indicators measured at baseline are given in Table 1. A detailed report on the methods applied has been published previously (31). Information on the vital status and migration of the participating inhabitants of Zoetermeer has been provided by the municipal authorities since the start of follow-up. Until May 1st 1987, the censor date, 490 members of the cohort $(7.5 \%)$ were lost to follow-up due to migration, and because the dates of migration were unknown they were excluded from the analysis. The remaining 2,839 men and 3,218 women were included in the present study.

\section{Data analysis}

Because the follow-up period varied between the participants, the proportional hazards model was applied to assess the cardiovascular risk indicators measured at baseline that were determinants of allcause mortality (Appendix) $(2,5)$. The proportionality assumption was checked using the log minus log survival function for the different variates (13). First, a bivariate analysis was performed, taking differences in age into account. Second, a multivariate model was applied in order to control for confounding. Only risk indicators that showed a clear association with mortality as indicated by the bivariate analysis (i.e., the coefficient/standard error $\geq 1.5$ ), were included in the multivariate model. Identical models were used for men and women.

TABLE 1. - Cardiovascular risk indicators measured at baseline.

\begin{tabular}{ll} 
Risk indicator & Measurement and/or units \\
\hline age & years \\
body mass index & weight/lenght ${ }^{2}$ in $\mathrm{kg} / \mathrm{m}^{2}$ \\
blood pressure & $\mathrm{mm} \mathrm{Hg}$, mean of two consecutive measurements with a random zero \\
sphygmomanometer (diastolic blood pressure based on Korotkoff 5) \\
beats/minute \\
pulse rate & mmol/l, method of Roesch-Lau \\
serum cholesterol & mmol/1, method of Gochman and Schmitz \\
serum uric acid & mmol/1, Technicon method \\
serum creatinine & current smoking (yes/no) \\
cigarette use & number of cigarettes/day smoked presently \\
& current coffee use (yes/no) \\
coffee use & number of cups/day used presently \\
for the indication hypertension (yes/no) & insulin or oral hypoglycemic drug use (yes/no) \\
antihypertensive drugs & history of myocardial infarction (yes/no) \\
myocardial infarction & history of stroke (yes/no) \\
stroke & premenopausal/postmenopausal \\
menopausal state & current use (yes/no) \\
oral contraceptives &
\end{tabular}


To estimate an individual's probability of dying within a certain time span as a function of the level of the cardiovascular risk indicators, risk functions were obtained (Appendix). A risk indicator was included in the formula when it was a predictor of mortality in the multivariate analysis in either sex, i.e., a 95\% confidence interval of the estimated relative risk not including 1.0. Separate risk functions were derived for men and women. The goodness of fit of the risk functions was checked computing the statistic $\mathrm{C}^{*} \mathrm{~g}$ (Appendix) (17).

\section{RESULTS}

\section{Determinants of all-cause mortality}

The mean value or prevalence of the cardiovascular risk indicators measured at baseline are shown in Table 2. In the 9 to 12 year follow-up period $10.8 \%$ of the men and $8.4 \%$ of the women died.

Cardiovascular determinants showing an ageadjusted association with mortality were systolic blood pressure, diabetes mellitus and a history of myocardial infarction in both sexes; diastolic blood pressure, pulse rate, smoking habits, antihypertensive drug use and a history of stroke especially in men; and body mass index (BMI), serum creatinine and uric acid most pronounced in women. Serum cholesterol, coffee use, menopausal state and use of oral contraceptives were not related to all-cause mortality in the bivariate analysis.
Because systolic blood pressure was a more powerful predictor of all-cause mortality than diastolic blood pressure, the former was included in the multivariate model. Of the two indicators for smoking habits, only the one reflecting current smoking (yes/no) was used.

The results of the multivariate analyses are shown in Table 3. The relative risks (RR) represent estimates of mortality risk of the presence of a risk indicator relative to its absence, or of the increase in its level by the indicated magnitude. Multivariate analysis did not alter the main findings of the age-adjusted analysis in men. In women, the impact of serum creatinine, uric acid and a history of myocardial infarction on survival weakened. An increase in BMI, however, remained strongly and inversely related to mortality.

In order to further investigate the apparent protective effect of an increasing BMI, the participants were categorized according to quartiles of BMI. The relative risk by quartiles of BMI is shown in Figure 1. A gradual risk reduction with increasing BMI is present in women only (test for trend in men: $p>0.10$; test for trend in women: $p<0.05)(29)$. The risk of death for women in the highest quartile of BMI relative to those in the lowest quartile was 0.56 (95\% confidence limits 0.36 and 0.87 ). Smoking status had a clear effect on allcause mortality in men only. To study the presence of a dose-response relationship, participants were categorized according to number of cigarettes smoked per day. A dose-response relationship was present in men, but not in women (Fig. 2).

TABLE 2. - Mean values and standard deviations (SD) or prevalence (\%) of risk indicators measured at baseline.

\begin{tabular}{lcccc}
\hline Risk indicator & \multicolumn{2}{c}{$\begin{array}{c}\text { men } \\
\text { mean (SD) } \\
\text { or \% }\end{array}$} & $\mathrm{n}^{*}$ & $\begin{array}{c}\text { women } \\
\text { mean (SD) } \\
\text { or \% }\end{array}$ \\
\hline age (years) & 2839 & $45.1(14.7)$ & 3218 & $46.6(16.0)$ \\
body mass index (kg/m²) & 2775 & $24.4(2.9)$ & 3167 & $24.4(3.8)$ \\
systolic bp (mm Hg) & 2782 & $132.1(17.5)$ & 3172 & $129.4(20.8)$ \\
diastolic bp (mm Hg) & 2782 & $79.1(11.9)$ & 3172 & $78.4(12.0)$ \\
pulse rate (beats/min) & 2779 & $75.8(13.0)$ & 3165 & $78.8(13.3)$ \\
serum cholesterol (mmol/1) & 2769 & $5.8(1.1)$ & 3154 & $5.8(1.1)$ \\
serum uric acid (mmol/1) & 2767 & $0.3(0.1)$ & 3154 & $0.3(0.1)$ \\
serum creatinine (umol/1) & 2767 & $91.4(15.7)$ & 3154 & $77.8(14.4)$ \\
current cigarette use & 2773 & $52.7 \%$ & 3186 & $38.2 \%$ \\
cigarettes per day** & 1460 & $15.6(8.2)$ & 1214 & $11.4(8.0)$ \\
current coffee use & 2832 & $97.7 \%$ & 3215 & $97.1 \%$ \\
cups of coffee per day & 2832 & $5.2(2.4)$ & 3215 & $4.2(2.1)$ \\
antihypertensive drugs & 2839 & $7.0 \%$ & 3218 & $16.2 \%$ \\
diabetes mellitus & 2718 & $1.1 \%$ & 3120 & $1.2 \%$ \\
myocardial infarction & 2835 & $3.1 \%$ & 3213 & $1.6 \%$ \\
stroke & 2835 & $0.6 \%$ & 3213 & $0.6 \%$ \\
postmenopausal & -- & -- & 3198 & $39.9 \%$ \\
oral contraceptive use & -- & -- & 3177 & $23.0 \%$ \\
\hline
\end{tabular}

* The numbers (n) given in the table correspond to the total number of men and women for whom data on a risk indicator were obtained.

** Non-smokers excluded.

$\mathrm{bp}=$ blood pressure. 
TABLE 3. - Cardiovascular risk indicators and all-cause mortality. Relative risk (RR) and 95\% confidence interval $(95 \% \mathrm{CI})^{*}$.

\begin{tabular}{lcccc}
\hline Risk indicator & \multicolumn{2}{c}{$\begin{array}{c}\text { men } \\
(\mathrm{n}=2624)^{* *} \\
(95 \% \mathrm{CI})\end{array}$} & $\mathrm{RR}$ & $\begin{array}{c}\text { women } \\
(\mathrm{n}=3048)^{* *} \\
(95 \% \mathrm{CI})\end{array}$ \\
\hline age (per 5 years) & 1.61 & $(1.53-1.70)$ & 1.49 & $(1.41-1.58)$ \\
body mass index (per $\left.3 \mathrm{~kg} / \mathrm{m}^{2}\right)$ & 0.92 & $(0.81-1.05)$ & 0.88 & $(0.79-0.97)$ \\
systolic blood pressure (per 10 mmHg) & 1.04 & $(0.99-1.11)$ & 1.09 & $(1.02-1.15)$ \\
pulse rate (per 10 beats/minute) & 1.10 & $(1.01-1.20)$ & 1.02 & $(0.93-1.13)$ \\
serum uric acid (per 0.1 mmol/l) & 1.04 & $(0.88-1.22)$ & 1.17 & $(0.98-1.40)$ \\
serum creatinine (per 20 $\mathrm{mmol} / \mathrm{l})$ & 1.03 & $(0.89-1.20)$ & 1.11 & $(0.95-1.29)$ \\
current cigarette use (yes/no) & 1.55 & $(1.20-1.99)$ & 0.89 & $(0.62-1.28)$ \\
antihypertensive drugs (yes/no) & 1.42 & $(1.02-1.96)$ & 1.06 & $(0.78-1.42)$ \\
diabetes mellitus (yes/no) & 1.86 & $(1.08-3.19)$ & 2.15 & $(1.21-3.82)$ \\
myocardial infarction (yes/no) & 1.92 & $(1.30-2.83)$ & 1.46 & $(0.90-2.36)$ \\
stroke (yes/no) & 1.19 & $(0.51-2.74)$ & 1.39 & $(0.51-3.79)$ \\
\hline
\end{tabular}

* Adjusted for differences in the other risk indicators.

** The number of men and women corresponds to the number of subjects for whom all variables considered in the multivariate analysis were measured.

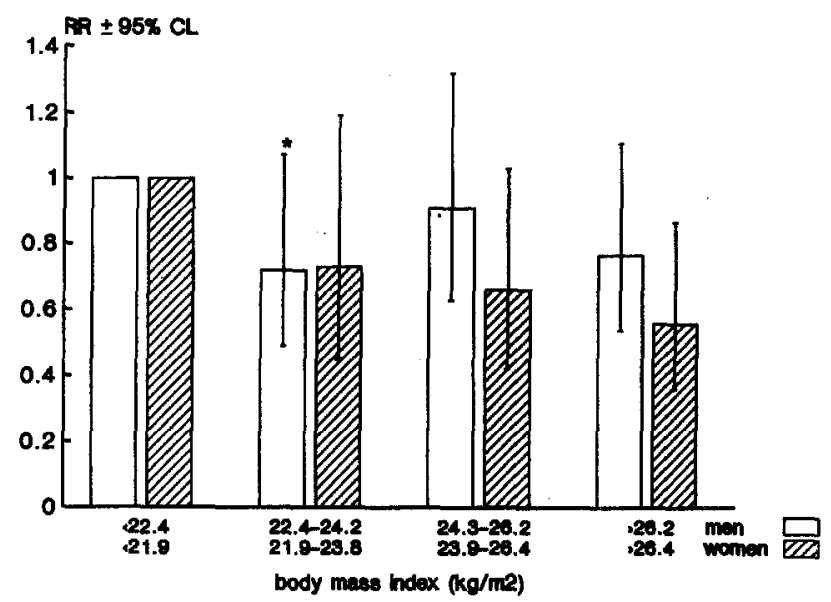

Figure 1. - Body mass index (BMI) and all-cause mortality in men and women. Relative risk (RR) and $95 \%$ confidence limits $(95 \% \mathrm{CL})^{*}$ of the four quartiles of BMI; the lowest quartile serves as a reference group $(R R=1)$. Adjusted for differences in the other risk indicators.

\section{Risk function}

Determinants that were independently associated with overall mortality in either sex were included in the risk function. Risk functions predicting the probability of dying within 11.5 years were calculated (Appendix). The sex-specific coefficients of the cardiovascular risk indicators included in the risk functions are shown in Table 4.

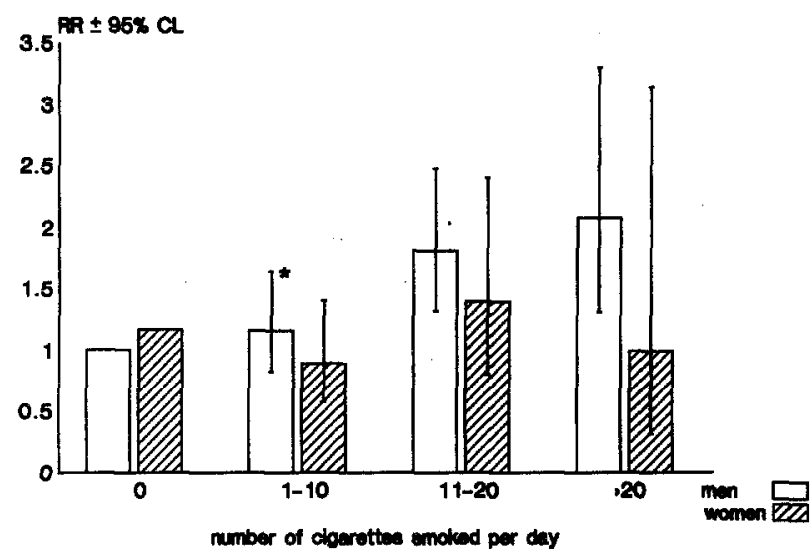

Figure 2. - Smoking habits and all-cause mortality in men and women. Relative risk (RR) and $95 \%$ confidence limits $(95 \% \mathrm{CL})^{*}$ of the number of cigarettes smoked per day; non-smokers serve as the reference group $(R R=1)$. Adjusted for differences in the other risk indicators.

The expected and observed number of male survivors and deaths within 11.5 years, for each decile of mortality probability are given in Table 5. The goodness of fit statistic equals $6.8(0.50<p<0.75)$. The value for the corresponding risk function in women is $14.9(0.05<\mathrm{p}<0.1)$. This indicates that the model has a moderate fit in women and that the risk function predicts mortality in men very accurately. 
TABLE 4. - Cardiovascular risk indicators and all-cause mortality in men and women; coefficients ( $B$ ) and standard errors (SE) of the risk indicators included in the risk function (multivariate analysis).

\begin{tabular}{|c|c|c|c|c|c|}
\hline \multirow{2}{*}{ Risk indicator } & & \multicolumn{2}{|c|}{ B } & \multicolumn{2}{|c|}{ SE } \\
\hline & & women & men & women & men \\
\hline age (years) & B1 & 0.096 & 0.081 & 0.005 & 0.006 \\
\hline body mass index $\left(\mathrm{kg} / \mathrm{m}^{2}\right)$ & B2 & -0.033 & -0.036 & 0.022 & 0.017 \\
\hline systolic bp (mm Hg) & B3 & 0.004 & 0.010 & 0.003 & 0.003 \\
\hline pulse rate (beats/min) & B4 & 0.009 & 0.001 & 0.004 & 0.010 \\
\hline cigarette use & B5 & 0.414 & -0.119 & 0.129 & 0.186 \\
\hline antihypertensive drugs & B6 & 0.372 & 0.160 & 0.159 & 0.143 \\
\hline diabetes mellitus & B7 & 0.594 & 0.700 & 0.274 & 0.291 \\
\hline myocardial infarction & B8 & 0.672 & 0.436 & 0.193 & 0.246 \\
\hline
\end{tabular}

$\mathrm{bp}=$ blood pressure

TABLE 5. - Goodness of fit of the risk function estimating the probability of dying within 11.5 years in men.

\begin{tabular}{|c|c|c|c|c|c|}
\hline \multirow[t]{2}{*}{ Decile } & \multirow{2}{*}{$\begin{array}{c}\text { limits of risk } \\
\%\end{array}$} & \multicolumn{2}{|c|}{ number of deaths } & \multicolumn{2}{|c|}{ number of survivors } \\
\hline & & expected & observed & expected & observed \\
\hline 1 & $<0.83$ & 1.6 & 1 & 262.4 & 263 \\
\hline 2 & $0.83-1.30$ & 2.9 & 2 & 260.1 & 261 \\
\hline 3 & $1.31-1.96$ & 4.2 & 5 & 259.8 & 259 \\
\hline 4 & $1.97-2.91$ & 6.3 & 3 & 257.7 & 261 \\
\hline 5 & $2.92-4.00$ & 9.0 & 4 & 255.0 & 260 \\
\hline 6 & $4.01-6.04$ & 13.2 & 10 & 250.8 & 254 \\
\hline 7 & $6.05-9.02$ & 19.7 & 17 & 246.3 & 249 \\
\hline 8 & $9.03-14.95$ & 30.2 & 32 & 232.8 & 231 \\
\hline 9 & $14.96-30.17$ & 56.2 & 59 & 207.8 & 205 \\
\hline \multirow[t]{2}{*}{10} & $>30.17$ & 140.5 & 141 & 124.5 & 124 \\
\hline & Total & 283.8 & 274 & 2357.3 & 2367 \\
\hline
\end{tabular}

$\mathrm{C}^{*} \mathrm{~g}=\sum_{\mathrm{k}=0}^{\stackrel{1}{\Sigma}} \quad \sum_{\mathrm{l}=0}^{10} \quad\left(\mathrm{O}_{\mathrm{k} l}-\mathrm{E}_{\mathrm{k} 1}\right)^{2} / \mathrm{E}_{\mathrm{k} 1}=6.83 ; 0.50<\mathrm{p}<0.75$

\section{DISCUSSION}

The primary objective of the present analysis was to assess the impact of cardiovascular risk indicators on all-cause mortality. The rationale for this approach is the possibility that certain preventive measures may, while having a beneficial effect on cardiovascular mortality, simultaneously increase mortality from other (non-cardiovascular) causes and hence, have no effect on or even decrease life expectancy. Alternatively, some measures could prove to be effective in preventing several chronic conditions at the same time.

Most of the cardiovascular risk indicators that demonstrate an effect on overall mortality in the present analysis have been recognized in similar follow-up studies $(6,8,11,16,18,22,23,24,25,30)$. Nevertheless, some of our findings warrant further elaboration and will be discussed below.

The apparent "protective" effect of an increasing body mass index, especially in women, is a remarkable finding. In most other cohort studies either no effect 
was reported, or a $\mathbf{J}$ - or $\mathrm{U}$-shaped relationship between BMI and mortality was found $(7,9,10,21,22,23)$. When women were categorized according to quartiles of BMI a gradual decrease of the RR from 1.0, in the lowest BMI group, to 0.56 in the highest BMI group was present, without evidence of a J- or U-shaped curve. Several possible explanations of the inverse relationship between BMI and mortality were evaluated (21). Because hypertension, glucose intolerance and hyperlipidaemia have been reported to act as intermediate factors in the causal pathway through which obesity influences longevity, it could be argued that adjustment for these factors in multivariate analysis should be avoided when assessing the overall effect of BMI on mortality. As expected, exclusion of these "effects of obesity" from the model attenuated the inverse relationship between BMI and mortality, but the effect remained statistically significant. Also, exclusion of all participants with diabetes mellitus at the baseline examination did not influence the association between body mass index and death from all causes. Further, a protective effect of a larger BMI might result from excess mortality in the leanest subjects caused by an underlying condition, such as cancer, leading to weight loss and subsequently to premature death. Disregarding the first three years of follow-up, however, did not materially change the findings. Smoking status has been recognized as a strong confounder of the association between BMI and longevity (9). Adjustment for current smoking and the number of cigarettes smoked daily did not alter the reported association. Yet, when smoking and non-smoking women were analyzed separately, the effect appeared to be restricted to smoking women only. From this analysis, residual confounding by smoking can not be excluded as an explanation for the inverse association. However, our findings clearly do not support an increase in risk with increasing body mass index. Therefore, the categorical labeling of overweight as unhealthy might prove to be unjustified when the outcome is longevity.

No clear association between serum cholesterol level and all-cause mortality could be demonstrated, a finding similar to that in the Dutch male cohort of the Seven Countries Study (24), but at variance with some other large studies $(22,28)$. Moreover, no evidence was found of a J-shaped relationship between cholesterol level and mortality, nor could cholesterol be identified as a predictor of mortality within different age categories. Thus, contrary to the established role played by serum cholesterol in atherosclerotic vessel disease $(3,20)$, no similar importance for all-cause mortality could be detected in this study. This could be due to the size of our study, suggesting however that an effect, if present, is small.

No association between smoking habits and mortality was found in women. This was unexpected, since cigarette smoking is a well-known predictor of cancer and cardiovascular mortality in both sexes.
This phenomenon may result in part from a lower smoking rate in women than in men (11.4 and 15.6 cigarettes/day respectively), but the absence of a doseresponse relationship in women in contrast to men, remains unexplained.

The second objective of this study was to obtain functions that predict an individual's probability of dying within a certain time period conditional on the risk profile. Since the Framingham cardiovascular risk function was published in 1976 (14), the use of such formulas has played a modest but increasing role in medical practice (1). Although the risk functions calculated from our study predict mortality satisfactory, as indicated by the goodness of fit statistic, the predictive value in men is better than in women. This might be explained in part by the lower mortality rate in women compared to men, leading to more accurate estimates of the coefficients in the latter group.

Several limitations of our study need to be discussed. It has been suggested that selective response in follow-up studies can lead to a relatively healthy cohort caused by an overrepresentation of participants at low risk. This selection bias seems to be limited in our study, since mortality rates among male and female participants (10.8 and $8.3 / 1000$ person years, respectively) are similar to the age-adjusted rates in the Dutch population at large (11.8 and 7.4/1000 person years, respectively) (4). Nevertheless, this effect could be diluted by a selective loss to follow-up of the healthy members of the cohort. Some evidence of the latter exists in that the cardiovascular risk profile of the 490 participants who were lost to follow-up due to migration was more beneficial than that of the remaining members, as indicated by a lower mean age (35.2 years in men, 37.0 years in women) and a lower systolic blood pressure $(130.3 \mathrm{~mm} \mathrm{Hg}$ in men, 124.6 $\mathrm{mm} \mathrm{Hg}$ in women). To further study the representativeness of our study population, the prevalence of the major cardiovascular risk indicators in our sample was compared with the prevalence reported in other studies performed in the Netherlands. The reported prevalence of most risk indicators, e.g. diabetes mellitus, hypertension and smoking, was similar to our findings $(31,32)$.

All risk indicators were measured only once at the start of the study. Hence, changes in the risk profile within the follow-up period could not be taken into account (12).

Finally, it must be stressed that the results of an analysis of determinants of all-cause mortality can only be applied to populations with similar patterns of causes of death. The causes of death among the 576 participants who died during the follow-up period were comparable to the causes of death recorded in all of the Netherlands from 1980 to 1984. For example, cardiovascular diseases accounted for $42 \%$ of all deaths in our cohort, while the corresponding proportion for the Netherlands was 43\% (19).

In summary, our findings suggest that the major cardiovascular risk indicators not only affect 
cardiovascular morbidity and mortality, but also influence survival irrespective of cause of death. By inference, favourable changes in these characteristics might lead to increase in life expectancy. The maximum individual benefit to be expected from these changes can be estimated using the risk functions derived from our data.

\section{Acknowledgements}

This work was supported by grants from the Netherlands Heart Foundation and the Netherlands Prevention Fund.

\section{APPENDIX}

The proportional hazards model is based on the hazard function:

$$
\lambda(\mathrm{t}, \mathrm{z})=\lambda_{0}(\mathrm{t}) \cdot \exp (ß 1 . z 1+\beta 2 . z 2+\ldots \beta \mathrm{p} . \mathrm{zp}) .
$$

The corresponding survival function is given by:

$$
\mathrm{S}(\mathrm{t}, \mathrm{z})=\mathrm{S}_{0}(\mathrm{t})^{\exp (\mathrm{B} 1 . \mathrm{z} 1+B 2 . z 2+\ldots B \mathrm{~B} . \mathrm{zp})}
$$

These formulas describe the hazard rate $\lambda(t, z)$ or probability of survival $S(t, z)$ as being dependent on the basic hazard rate $\lambda_{0}(t)$ or basic survival function $S_{0}(t)$ respectively, and on a risk indicator function ( $B 1 . z 1+\ldots B p . z p)$. The coefficients $B 1$ to $B p$ are estimated by the computer program (2), and $\mathrm{zl}$ to $\mathrm{zp}$ are an individual's level of the risk factors. The basic hazard rate and survival function correspond to the incidence rate and the survival probability for individuals with (extrapolated) levels of the risk indicators of zero, and thus only changes with time elapsed since the start of the follow-up period.

A risk function takes the following form in the proportional hazards model:

$$
\mathrm{P}(\mathrm{t})=1-\mathrm{S}(\mathrm{t}, \mathrm{z})=1-\mathrm{S}_{0}(\mathrm{t})^{\exp (B 1 . z 1+B 2 . z 2+\ldots B \mathrm{p} . \mathrm{zp})}
$$

$P(t)$ indicates the probability of an event within a certain period and equals 1 - $S(t, z)$. The formula includes a time-dependent variate $S_{0}(t)$, which is not provided by the standard computer programs applying the model. In the present analysis the values of $S_{0}(t)$ were calculated indirectly using an option in the BMD P2L program which gives a table containing the survival probability as a function of time for the mean vector, i.e., an individual with all risk indicators equal to the population mean (2).

The goodness of fit of the risk function can be assessed by computing the statistical characteristic $\mathrm{C}^{*} \mathrm{~g}$ as proposed by Lemeshow and Hosmer (17):

$$
\mathrm{C}^{*} \mathrm{~g}=\begin{array}{cc}
1 & 10 \\
\sum & \sum \\
\mathrm{k}=0 & 1=0
\end{array} \quad\left(0_{\mathrm{k} 1}-\mathrm{E}_{\mathrm{kl}}\right)^{2} / \mathrm{E}_{\mathrm{kj}}
$$

where $O_{k 1}$ and $E_{k !}$ are the observed and expected number of deaths in the twenty cells formed by the deciles of risk ( 1 from 1 to 10$)$ for those with $(\mathrm{k}=1)$ or without $(\mathrm{k}=0)$ the end-point at interest. The distribution of the statistic is closely approximated by a chi-square distribution with (1 minus 2 ) degrees of freedom. The probability of dying within 11.5 years, the maximum followup period, for men equals: $1-0.99968^{\exp (\text { risk score). }}$

The probability of dying within 11.5 years for women equals: $1-0.99945^{\text {exp(risk score) }}$.

The individual risk scores in the formulas can be computed using the sex-specific coefficients $(B 1, B 2, \ldots B 8)$ in Table 4 and the actual level of the risk indicators in the following formula: risk score $=B 1$. age $+B 2$. body mass index $+B 3$. systolic blood pressure $+B 4$. pulse rate $+B 5$. cigarette use $($ yes $=1$, no $=0)+$ B6. antihypertensive drug use (yes $=1$, no $=0$ ) $+B 7$. diabetes $($ yes $=1$, no $=0)+B 8$. myocardial infarction (yes $=1$, no $=0$ ).

\section{REFERENCES}

1. Anderson K.M., Wilson P.W.F., Odell P.M. and Kannel W.B. (1991): An updated coronary risk profile. A statement for health professionals - Circulation 83 : 357-363.

2. BMDP Statistical Software (1988): University of California Press, Berkeley.

3. Castelli W.P., Garrison R.J., Wilson P.W.F., Abbott R.D., Kalousdian S. and Kannel W.B. (1986): Incidence of coronary heart disease and lipoprotein cholesterol levels: The Framingham Study J.A.M.A. 256: 2835-2838.

4. Central Bureau of Statistics (1986): Compendium of Health Statistics in the Netherlands 1986 Staatsuitgeverij, The Hague.

5. Cox D.R. (1972): Regression models and life tables J.R. Statis. Soc. B34: 187-220.

6. Dyer A.R., Perskey V., Stamler J., et al. (1980): Heart rate as a prognostic factor for coronary heart disease and mortality: Findings in three Chicago epidemiologic studies - Am. J. Epidemiol. 112: 736749.

7. Dyer A.R., Stamler J., Berkson D.M. and Lindberg H.A. (1975): Relationship of relative weight and body mass index to 14-year mortality in the Chicago People Gas Company Study - J. Chron. Dis. 28: 109-123.

8. Farchi G., Menotti $A$. and Conti S. (1987): Coronary risk factors and survival probability from coronary and other causes of death - Am. J. Epidemiol. 126: 400-408.

9. Garrison R.J., Feinleib M., Castelli W.B., et al. (1983): Cigarette smoking as a confounder of relative weight and long-term mortality: The Framingham Heart Study - J.A.M.A. 249: 2199-2203.

10. Gordon T. and Doyle J.T. (1988): Weight and mortality in men: The Albany Study - Int. J. Epidemiol. 17: 77-81.

11. Grobbee D.E. (1988): The rhythm and the risk: Heart rate and cardiovascular disease - Curr. Opinion. Cardiol. 3 (suppl. 3): S15-S21.

12. Hofman A., Feinleib M., Garrison M., Garrison R. and Van Laar A. (1983): Does change in blood pressure predict heart disease? - Br. Med. J. 287: 267-269.

13. Kalbfleish J.D. and Prentice R.L. (1980): The statistical analysis of failure time data - John Wiley \& Sons, New York.

14. Kannel W.B., McGee D. and Gordon T. (1976): A general cardiovascular risk profile: The Framingham Study - Am. J. Cardiol. 38: 46-51.

15. Keys A., Aravanis C., Blackburn H., et al. (1980): Seven Countries. A multivariate analysis of death and coronary heart disease - Harvard University Press, Cambridge. 
16. Keys A., Menotti A., Aravanis A., et al. (1984): The Seven Countries Study: 2,289 deaths in 15 years Prev. Med. 13: 141-154.

17. Lemeshow S., Hosmer Jr D.W. (1982): A review of goodness of fit statistics for use in the development of logistic regression models - Am. J. Epidemiol. 115: 92-106.

18. Levine W., Dyer A.R., Shekelle R.B., Schoenberger J.A. and Stamler J. (1989): Serum uric acid and 11.5-year mortality of middle-aged women: Findings of the Chicago Heart Association Detection Project in Industry - J. Clin. Epidemiol. 42: 257-267.

19. Mackenbach J.P. (1988): Mortality and medical care Thesis, Erasmus University, Rotterdam.

20. Manolio T.A., Pearson T.A., Wenger N.K., BarrettConnor E., Payne G.H. and Harlan W.R. (1992): Cholesterol and heart disease in older persons and women. Review of an NHBLI Workshop - Ann. Epidemiol. 2: 161-176.

21. Manson J.E., Stampfer M.J., Hennekens C.H. and Willett W.C. (1987): Body weight and longevity: A reassessment - J.A.M.A. 257: 353-358.

22. Martin M., Browner W.S., Wentworth D., Hulley S.B. and Kuller L.H. (1986): Serum cholesterol, blood pressure, and mortality: Implications from a cohort of 361,662 men - Lancet ii: 923-926.

23. Menotti A., Conti S., Dima F., et al. (1983): Prediction of all causes of death as a function of some factors commonly measured in cardiovascular population surveys - Prev. Med. 12: 318-325.

24. Menotti A., Keys A., Nissinen A., Kromhout D. and Seccareccia F. (1988): The Seven Countries Study. 25 years experience in three countries. In: Proceedings of the 8th International Symposium on Atherosclerosis: Epidemiology of Atherosclerosis (Satellite Meeting) - Porto Cervo pp. 19-25.

25. Menotti A., Mariotti S., Seccareccia F., Torsello S. and Dima F. (1987): Determinants of all causes of death in samples of Italian middle-aged men followed up for 25 years - J. Epidemiol. Comm. Health 41: 243-250.
26. Neaton J.D. and Wentworth D. (1992): Serum cholesterol, blood pressure, cigarette smoking, and death from coronary heart disease. Overall findings and differences by age for 316,099 white men - Arch. Intern. Med. 152: 56-64.

27. Pooling Project Research Group (1978): Relationship of blood pressure, serum cholesterol, smoking habits, relative weight and ECG abnormalities to incidence of major coronary events. Final report of the Pooling Project - J. Chron. Dis. 31: 201-306.

28. Rose G. and Shipley M.J. (1980): Plasma lipids and mortality: A source of error - Lancet $i$ : 523-526.

29. Rothman K.J. (1986): Modern Epidemiology - Little, Brown \& Co., Boston.

30. Shurtleff $D$. (1974): Some characteristics related to the incidence of cardiovascular disease and death. In: Kannel W.B., Gordon T., Eds. Framingham study. An epidemiological investigation of cardiovascular disease - Section 30 DHEW Publication (NHI) No 74-599.

31. Valkenburg H.A., Hofman A., Klein F. and Groustra F.N. (1980): An epidemiological study of cardiovascular risk indicators (EPOZ). I. Blood pressure, serum cholesterol level, Quetelet index and smoking habits in an open population aged five years and over - Ned Tijdschr Geneeskd 124: 183-189.

32. Van de Lisdonk E.H., Van den Bosch W.J.H.M., Huygen F.J.A., Lagro-Janssen A.L.M., Eds. (1990): Ziekten in de huisartspraktijk (Morbidity in general practice) - Wetenschappelijke Uitgeverij Bunge, Utrecht.

33. Wannamethee G. and Shaper A.G. (1989): Body weight and mortality in middle aged British men: Impact of smoking - Br. Med. J. 299: 1497-1502.

34. Wilhelmsen L., Wedel H. and Tibblin G. (1973): Multivariate analysis of risk factors for coronary heart disease - Circulation 48: 950-958. 